\title{
Fall point probability density model based on Monte Carlo
}

\author{
Rui Ma \\ School of North China Electric Power University Baoding, Baoding 071000, China; \\ 1150229173@qq.com
}

Keywords: probability density lost plane Monte Carlo

\begin{abstract}
Searching a lost plane in open water is not an easy thing as we thought. To make the searching working more efficient, we build fall point probability density model to find a possible searching area of the lost plane. In our model, we first obtain an equation set about the position coordinate of the crashed plane. Then based on the Monte Carlo algorithm, we simulate 10000 points and get the distribution of the probability density of falling point. We also make it clear of different types of lost planes and discuss the influence of the cruising altitude and lift drag ratio. We finally get the probability density of the falling point and find out the prior search area.
\end{abstract}

\section{Introduction}

The Malaysia Airlines flight MH370 has been lost for more than one year. Though many countries have sent planes and ships to search the plane, the location remains unclear. There are many difficulties of searching a lost plane in open water. Firstly, the contact between plane and ground control center is not continuous, thus it is difficult to get accurate information of the crash point. Next, the scene of the crash is changing every moment. The debris will be broke up and drift with the ocean currents and waves, which enhance the difficulties of determining the scope of the search. Last but not least, power on the black box of the plane works only 30-day time, so salvage must race against time.

There are many different types of lost planes. Considering the impact on the plane crashed, these differences can be attributed to two aspects: cruising altitude of the plane and the lift drag ratio. Also, the differences among searching planes mainly lie in the voyage, the searching view and reliability.

After the above two points have been made clear, we establish a fall point probability density model to simulate the process of falling. Based on the Monte Carlo algorithm, we get the probability density of the falling point and find out the prior search area.

\section{The Method}

To search a lost plane efficiently, our first step is to determine an objective search area. In our fall point probability density model, we simulate the falling process of the plane to give a possible area.

Our major assumption is that the plane can be regarded as a particle before it touches the surface of the water. About different types of planes for which we might be searching, we think the main differences lie in two aspects: cruising altitude and the lift drag ratio.

On the basis of the assumptions, we divide the falling process into two parts: deviation period and the falling period. It is unlikely that the plane can fly along the original track when out of control, so we define as the angle of the plane off course. According to relevant data information, we give a possible distribution for the value of . After deviation period, the plane will drop with angle at the falling period. We then derive an expression for the crash site .Next, based on the Monte-Carlo algorithm; we give the probability density distribution of the falling location.

On the cruise, the cruising altitudeof the plane is constant and we can get this information from the last contact before the crash. Considering the angle between the falling direction and the horizontal direction, $d$ can be represented as: 
As we assumed before, the optimal driving strategy for pilot is to make sure the is minimum. We find the minimum value of is: ${ }^{[1]}$

In the formula above is the lift drag ratio of the plane, and it is affected by many factors, such as: aircraft angle of attack, flight speed and so on. In the next part, we will give the reference values of with different types of the plane.

Then we have the maximum distance between the lost location and the drop location:

Considering that if the power system of the plane is interrupted, the plane may lose control or the pilot may make decisions to change the flying direction. In reality, there is actually little chance for plane to fly along the original route. We let denotethe angle of the plane off course, the flight path is shown in Figure 1.

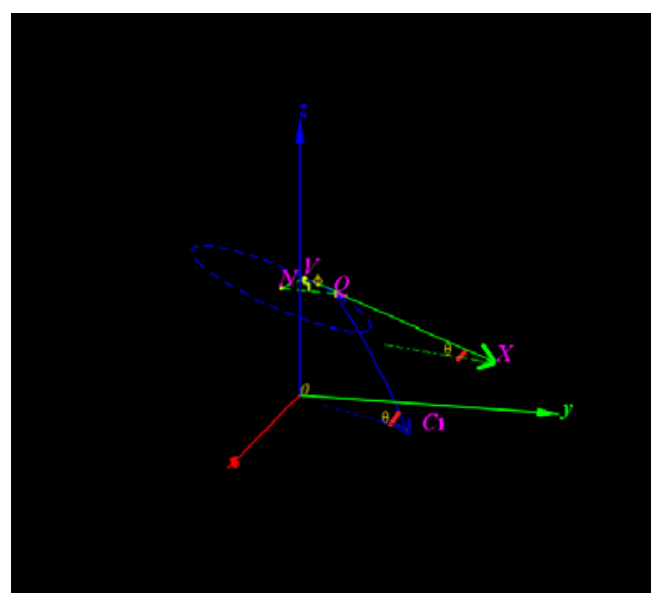

Figure 1. Flight orbit in the falling process

$V$ is the point where the plane begins to fall. The process from $V$ to $Q$ represents the deviation period and Nis the center of turning circle. The falling point in theory is C1.

According to the possible value of (), the falling point can be determined. Through the following coordinate transformation, we convert () into Descartes Cartesian coordinates:

We determine values for some parameters.

Different types of plane have different lift drag ratio. For general aircraft, lift drag ratio of low hormone and subsonic plane can up to 17 to 18 , transonic plane is up to 10 to 12 , supersonic aircraft with Mach number two is about 4-8. ${ }^{[2]}$ Thus we generally assume $=17$.

The cruising altitude $h$ of the plane varies due to the difference of the plane. We assume the cruising altitude $h$ to be 10668 meters.

Table 1. Flight parameters

\begin{tabular}{llll}
\hline Aircraft type & $\begin{array}{l}\text { Cruising } \\
\text { altitude }(\mathrm{m})\end{array}$ & $\begin{array}{l}\text { Maximum } \\
\text { range }(\mathrm{km})\end{array}$ & $\begin{array}{l}\text { Maximum flying } \\
\text { speed }(\mathrm{km} / \mathrm{h})\end{array}$ \\
\hline Boeing 737 & 10670 & 5890 & 831 \\
Boeing 747 & 10670 & 13570 & 956 \\
Boeing 757 & 11000 & 6426 & 830 \\
Boeing 767 & 13137 & 11305 & 851 \\
Boeing 777 & 11000 & 9695 & 830 \\
\hline
\end{tabular}

The coefficient $r$ is the turning radius of the plane, and isthe radius of curvature. On the basis of the central limit theorem, the employs the normal distribution with zero mean and variance of 36 . Also, the angle employs the normal distribution. We conservatively assume the mean values to be zero and variance to be900.

Based on Monte Carlo algorithm, we generate ten thousand groups of data randomly in the given range and get probability density distributing diagrams. Figure 2 shows the area where the plane most likely crashed. 


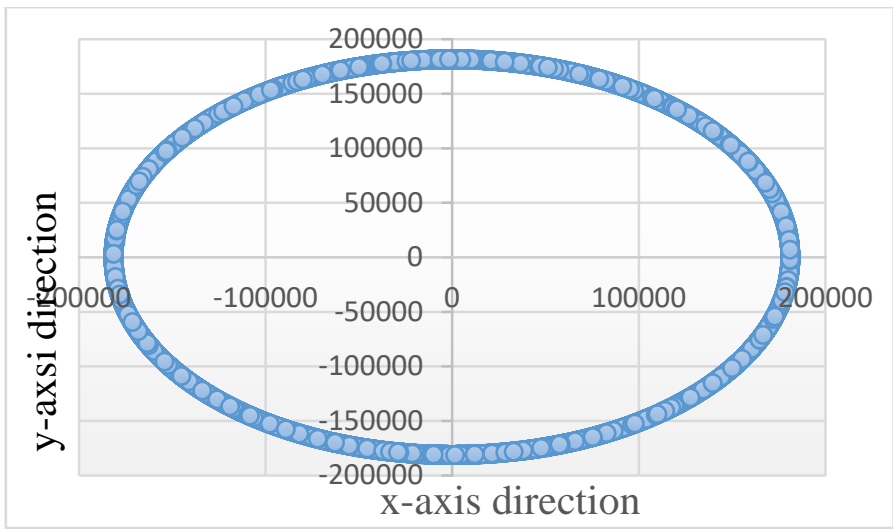

Figure 2. Possible area of the falling plane

\section{Summary}

We build fall point probability density model to make the searching work more efficient and obtain an equation set about the position coordinate of the crashed plane. Based on Monte Carlo algorithm, we generate ten thousand groups of data randomly in the given range and get probability density distributing diagrams and get the area where the plane most likely crashed as Figure 2 shows

\section{References}

[1]Anderson J D. Introduction to flight [M].Boston; McGraw-Hill, 2005.

[2]Lift drag ratio. (n.d.). Retrieved January 22, 2016, from http://baike.baidu.com/ [3]Aircraft Specifications. ( n. d.). Retrieved February 7, 2015, from http: // www. Gateway group .com / aircraft- specifications/

[4]Boeing 737.(n.d.). Retrieved February 6,2015, from http://en.wikipedia.org/wiki/Boeing_737

[5]Long,L,\&Weiss,H.(2006).How Terminal Is Terminal Velocity? The American Mathematical Monthly,(113),752-755. 